\title{
Étude du gonflement des sols pour des projets de tunnels
}

T. WINDAL

I. SHAHROUR

Laboratoire de mécanique de Lille

Université de Lille 1 EUDIL

Département génie civil

Cité scientifique avenue Paul-Langevin 59655 Villeneuve-d'Ascq Cedex timothee.windal@eudil.fr isam.shahrour@eudil.fr

\section{J.-P. MAGNAN}

Laboratoire central des ponts et chaussées

58 , bd Lefebvre

75732 Paris Cedex 15

magnan@lcpc.fr

\section{J.-F SERRATRICE}

CETE Méditerranée,

Laboratoire régional des ponts et chaussées

d'Aix-en-Provence BP 3700

13791 Aix-en-Provence Cedex 3

jean-francois.serratrice @equipement.gouv.fr
Cette communication porte sur l'étude du gonflement d'une marne " intacte » réalisée à l'aide d'un œdomètre flexible. Le dispositif utilisé autorise une certaine déformation latérale de l'éprouvette lors du gonflement et permet la mesure de la pression de gonflement exercée dans cette direction. La variation de la rigidité de l'œelomètre flexible permet d'étudier l'influence de la déformation du sol sur le gonflement et de reproduire les conditions rencontrées lors de la construction de tunnels flexibles dans des sols gonflants. Les essais réalisés montrent que la rigidité de l'anneau œdométrique ainsi que la stratification de l'éprouvette affectent sensiblement le gonflement.

Mots-clés : œdomètre flexible, gonflement, tridimensionnel, marne, pression latérale, tunnel, anisotropie, stratification.

\section{Study of soils swelling for tunnel projects}

This paper concerns a study of the swelling behavior of uintact 0 marl using a flexible odometer. This device allows for lateral deformation of the specimen during swelling process and permits the measurement of the lateral swelling pressure. The variation of stiffness of the odometer ring allows to study the influence of the soil deformation on swelling and to reproduce swelling condition encountered during the construction of flexible tunnels in swelling soils. Tests results show that the ring stiffness as well as the stratification of the specimen largely
} affect soil swelling.

Key words : flexible odometer, three-dimensional, swelling, marl, lateral pressure, tunnel, anisotropy, stratification. 


\section{Introduction}

Le gonflement de sols constitue un problème majeur pour les tunnels construits dans des terrains présentant un fort potentiel de gonflement (Einstein et Bischoff 1976, Steiner 1993, Kovari et al., 1995; Robert, 1991). Le suivi de nombreux tunnels montre que le gonflement induit un soulèvement du radier suivi par une dislocation et une rupture du revêtement. Steiner (1993) a recensé des pressions de gonflement variant de 0,15 à $0,7 \mathrm{MPa}$ pour des tunnels construits dans des schistes et des pressions plus importantes (de 2 à $2,5 \mathrm{MPa}$ ) pour les tunnels construits dans des matériaux contenant de l'anhydrite. Le phénomène de gonflement peut durer longtemps : à titre d'exemple, on peut citer le cas du tunnel du Upper-Hauenstein pour lequel on a mesuré une vitesse de soulèvement du radier de l'ordre de $10 \mathrm{~mm} /$ an 120 ans après sa construction (Robert, 1991).

Le calcul et la vérification de tunnels concernés par les sols gonflants nécessitent la détermination de la pression de gonflement induite dans les différentes directions. La caractérisation du gonflement est généralement effectuée à l'aide de l'œedomètre classique, qui impose une condition de déformation latérale nulle au cours du gonflement. Or, dans le cas des tunnels, et plus particulièrement ceux présentant une faible rigidité, on peut assister à une déformation latérale du sol au cours du processus de gonflement. Cette déformation peut influencer d'une manière sensible la pression de gonflement. Ce phénomène a été étudié dans la direction axiale par de nombreux auteurs en utilisant I'cedomètre classique (Holtz et Gibbs, 1956 ; Seed et al., 1962 ; Kabbaj, 1989). Leurs travaux ont montré que l'autorisation d'une faible déformation axiale réduit fortement la pression de gonflement dans cette direction.

On présente dans cet article les résultats d'une étude réalisée à l'aide d'un œdomètre flexible, du gonflement des marnes de l'agglomération marseillaise. Cet appareillage autorise une déformation latérale de l'éprouvette au cours du gonflement et permet la mesure de la pression de gonflement induite dans cette direction. Une description détaillée de l'appareillage est présentée par Windal (2001). La déformation latérale de l'éprouvette est contrôlée par la rigidité relative sol/celIule œedométrique. La variation de cette rigidité permet de réaliser des essais de gonflement allant de l'essai de gonflement libre (cellule très flexible) à l'essai cedométrique classique (cellule très rigide). Ces essais permettent d'étudier l'influence de la rigidité de l'anneau sur le gonflement et de disposer ainsi d'une base de données intéressantes pour la vérification de modèles de gonflement tridimensionnels (Wittke et Pierau, 1979; Froehlich, 1989 ; Kiehl, 1990; Bultel, 2001) ou le développement de nouveaux modèles pour le calcul de tunnels construits en terrains gonflants.

\section{2}

\section{Matériau étudié}

L'étude a été réalisée sur une marne argileuse prélevée par le Laboratoire régional des ponts et chaussées d'Aix-en-Provence, dans le cadre de la reconnaissance géotechnique d'un projet autoroutier situé dans l'agglomération marseillaise et destiné à relier les autoroutes $\mathrm{A} 7$ et $\mathrm{A} 55$. Des analyses minéralogiques semi- quantitatives ont été effectuées au LRPC d'Aix-en-Provence par diffraction $X$ sur des échantillons proches de ceux étudiés. Les tableaux la et Ib donnent la distribution des minéraux dans ce sol et le pourcentage des différentes argiles. On note un faible pourcentage de calcite et de quartz et une forte proportion d'argile. Les smectites prédominent largement, ce qui laisse présager un comportement gonflant pour ce matériau. Les échantillons ont été prélevés à une profondeur variant entre 35,85 et $36,20 \mathrm{~m}$. Ils ont une teneur en eau comprise entre 9,0 et $13,5 \%$. La détermination du poids volumique sec des matériaux proches permet d'encadrer le poids volumique sec des éprouvettes étudiées entre 18 et $20 \mathrm{kN} / \mathrm{m}^{3}$.

Les éprouvettes testées sont assez hétérogènes, on peut trouver au sein d'une même éprouvette des matériaux de couleurs assez variées (du gris clair au marron rouge en passant par le jaune). Certaines éprouvettes présentent une stratification plus ou moins marquée.

\section{3}

\section{Mode opératoire et programme expérimental}

Les essais de gonflement ont été réalisés selon la procédure d'Huder-Amberg à l'aide des cellules flexibles ayant des rigidités $\mathrm{K}_{\text {in }}=370,580,850$ et $3045 \mathrm{MPa}$. L'essai est réalisé en trois phases. La première phase consiste dans l'application d'un cycle de chargement-déchargement-rechargement jusqu'à une contrainte axiale $\sigma_{00}$ voisine de $780 \mathrm{kPa}$. Elle vise à soumettre l'éprouvette à la contrainte verticale correspondant à la contrainte in situ et à réduire le remaniement dû à l'extraction de l'éprouvette. La deuxième phase concerne l'imbibition de l'éprouvette jusqu'à la saturation sous la contrainte axiale $\sigma_{\text {to. }}$. Après stabilisation du gonflement, on effectue la troisième phase qui permet d'évaluer le potentiel de gonflement. Cette phase comporte un déchargement de l'éprouvette par palier pendant lequel on suit l'évolution de la déformation axiale et de la pression latérale jusqu'à leur stabilisation.

Deux essais ont été réalisés sur des éprouvettes non stratifiées avec les anneaux ayant des rigidités $\mathrm{K}_{\mathrm{an}}=850$ et $3045 \mathrm{MPa}$. Sur les éprouvettes stratifiées, nous avons réalisé trois essais avec les anneaux ayant les rigidités $\mathrm{K}_{\mathrm{m}}=370,580$ et $3045 \mathrm{MPa}$.

TABlEAU la. Distribution des minéraux dans la marne étudiée (Serratrice, 1998).

Distribution of minerals in studied marl (Serratrice, 1998),

\begin{tabular}{lcc} 
Calcite & Quartz & $\begin{array}{c}\text { Autres phases (argiles. } \\
\text { feldspath, dolomite) }\end{array}$ \\
\hline $21 \%$ & $10 \%$ & $69 \%$ \\
\hline
\end{tabular}

TABLEAU IO Pourcentage des argiles présentes dans la marne (Serratrice, 1998). Percentage of clays present in marl (Serratrice. 1998).

\begin{tabular}{lrrr} 
Smectite & llite & Kaolinite & Chlorites \\
\hline $65 \%$ & $28 \%$ & $5 \%$ & $2 \%$ \\
\hline
\end{tabular}




\section{Résultats et discussions}

On présente d'abord les résultats de la phase d'imbibition sous la contrainte axiale $\sigma_{00}=780 \mathrm{kPa}$; ensuite on donne les résultats obtenus lors de la phase de déchargement.

\section{1}

\section{Gonflement au cours de la phase d'imbibition}

On donne dans la figure 1 les résultats des essais réalisés avec l'anneau de rigidité élevée $\left(\mathrm{K}_{\text {n }}=\right.$ $3045 \mathrm{MPa}$ ) sur une éprouvette stratifiée et une éprouvette non stratifiée. On remarque que l'éprouvette stratifiée présente une déformation axiale de gonflement de l'ordre de $1,3 \%$, qui est supérieure de $50 \%$ au gonflement axial de l'éprouvette non stratifiée. En ce qui concerne la pression de gonflement latérale, on note que les deux éprouvettes développent des pressions latérales très proches (de l'ordre de $1050 \mathrm{kPa}$ ). Ces résultats indiquent clairement que la stratification des éprouvettes amplifie le gonflement axial, mais n'affecte que très légèrement le gonflement dans la direction latérale. Ce résultat est en bon accord avec les résultats des essais réalisés par Froehlich (1989) sur une roche stratifiée. On peut noter que la contrainte latérale induite par le gonflement $\left(\Delta \sigma_{i}\right)$ est supérieure à la contrainte axiale d'imbibition : ceci indique que le gonflement conduit l'éprouvette à un état d'extension $(\mathrm{q}=$ $\sigma_{\mathrm{a}}-\sigma_{1}<0$ ).

Les figures 2 et 3 illustrent respectivement les résultats des essais réalisés sur les éprouvettes stratifiées et non stratifiées avec les différents anneaux. Ces résultats montrent que l'augmentation de la rigidité de l'anneau induit une augmentation de la déformation axiale et de la pression de gonflement latérale. Pour les éprouvettes non stratifiées, l'augmentation de la rigi- dité d'anneau de $850 \mathrm{MPa}$ à $3045 \mathrm{MPa}$ induit un accroissement de l'ordre de $90 \%$ de la déformation axiale de gonflement et une augmentation de l'ordre de $210 \%$ de la pression latérale de gonflement (tableau II). Pour les éprouvettes stratifiées, on note que l'augmentation de la rigidité de l'anneau cedométrique de 370 $\mathrm{MPa}$ à $3045 \mathrm{MPa}$ induit un accroissement de $70 \%$ de la déformation axiale et une augmentation de $120 \%$ de la pression de gonflement latérale. Ces résultats montrent que le fait d'empêcher le gonflement dans la direction latérale a pour effet d'augmenter à la fois la pression de gonflement latérale et la déformation axiale de gonflement. Les valeurs du déviateur à la fin de la phase de gonflement libre sont reportées dans le tableau II. On peut remarquer que l'augmentation de la rigidité de la cellule réduit la valeur du déviateur à la fin de la phase d'imbibition et conduit l'éprouvette à un état d'extension pour les rigidités élevées.

\section{2}

\section{Phase de décharge : évaluation du potentiel de gonflement}

Les figures $4 a-d$ donnent les résultats de l'essai réalisé sur l'éprouvette stratifiée avec l'anneau de rigidité $\mathrm{K}_{\mathrm{an}}=3045 \mathrm{MPa}$. Pour chaque palier de déchargement, on observe une déformation instantanée suivie d'une augmentation de cette déformation avant sa stabilisation (Fig. 4a). Le déchargement induit également une réduction de la contrainte latérale suivie par une légère augmentation pour le premier palier (Fig. 4a). Les valeurs du rapport entre les variations de la contrainte latérale et de la contrainte axiale sont reportées dans le tableau III. On note que ce rapport est inférieur à 1 pour les deux premiers paliers. Ensuite il croît et devient supérieur à 1. Cette variation indique que les premiers paliers accentuent l'état d'extension de l'éprouvette (Fig. 4b).

La figure $4 c$ donne les résultats obtenus à la stabilisation dans le plan $\left(\log \sigma_{0},-\varepsilon_{n}\right)$. Les parties $(A-B-C-D)$,
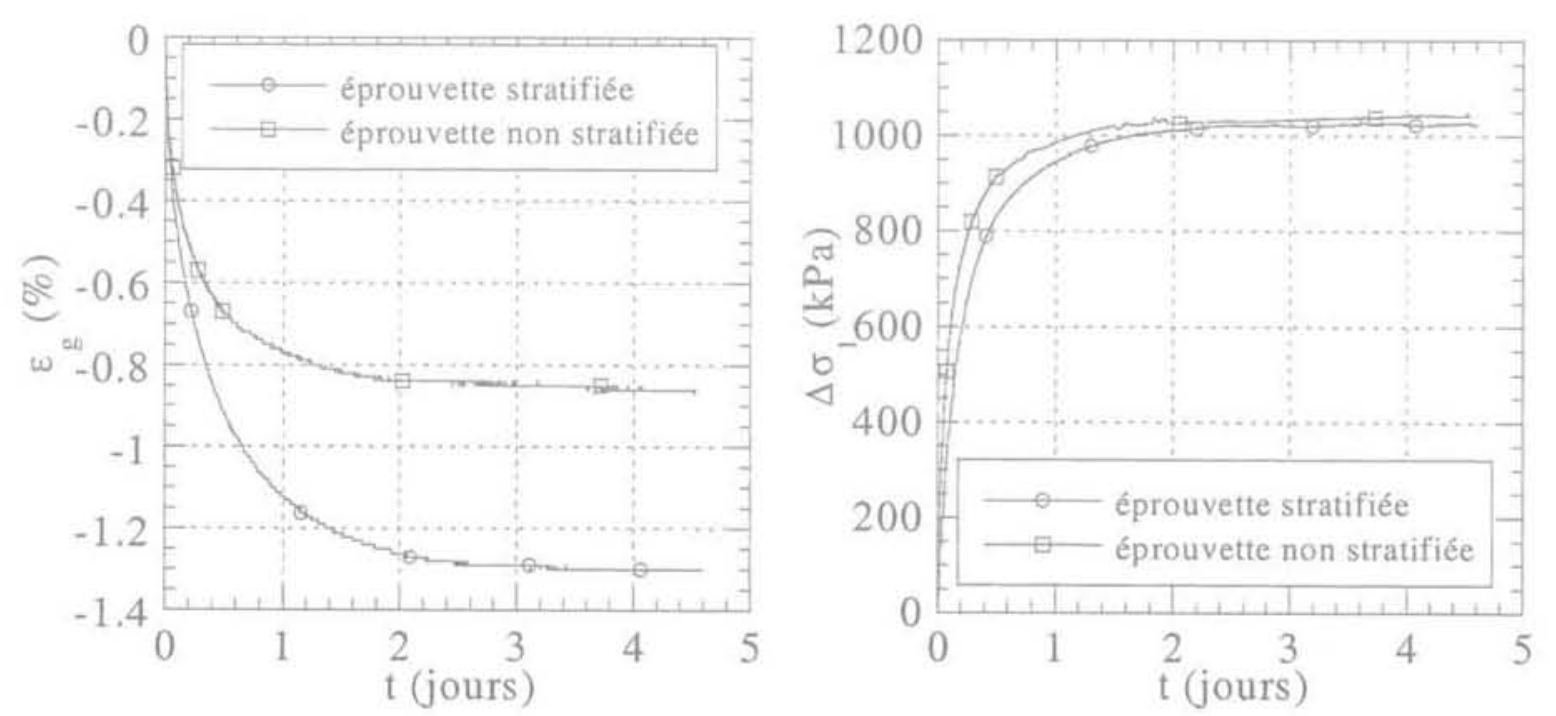

FIG. I Influence de la stratification sur le gonflement pendant la phase d'imbibition-essai réalisé avec l'anneau de rigidité $\mathrm{K}_{\mathrm{an}}=3045 \mathrm{MPa}$.

(a) Déformation de gonflement axiale ; (b) pression de gonflement latérale.

Influence of the stratification on swelling during the imbibition phase - test carried out with the ring stiffness $\mathrm{K}_{\text {an }}=3,045 \mathrm{MPa}$.

(a) Axial swelling strain; (b) lateral swelling pressure. 

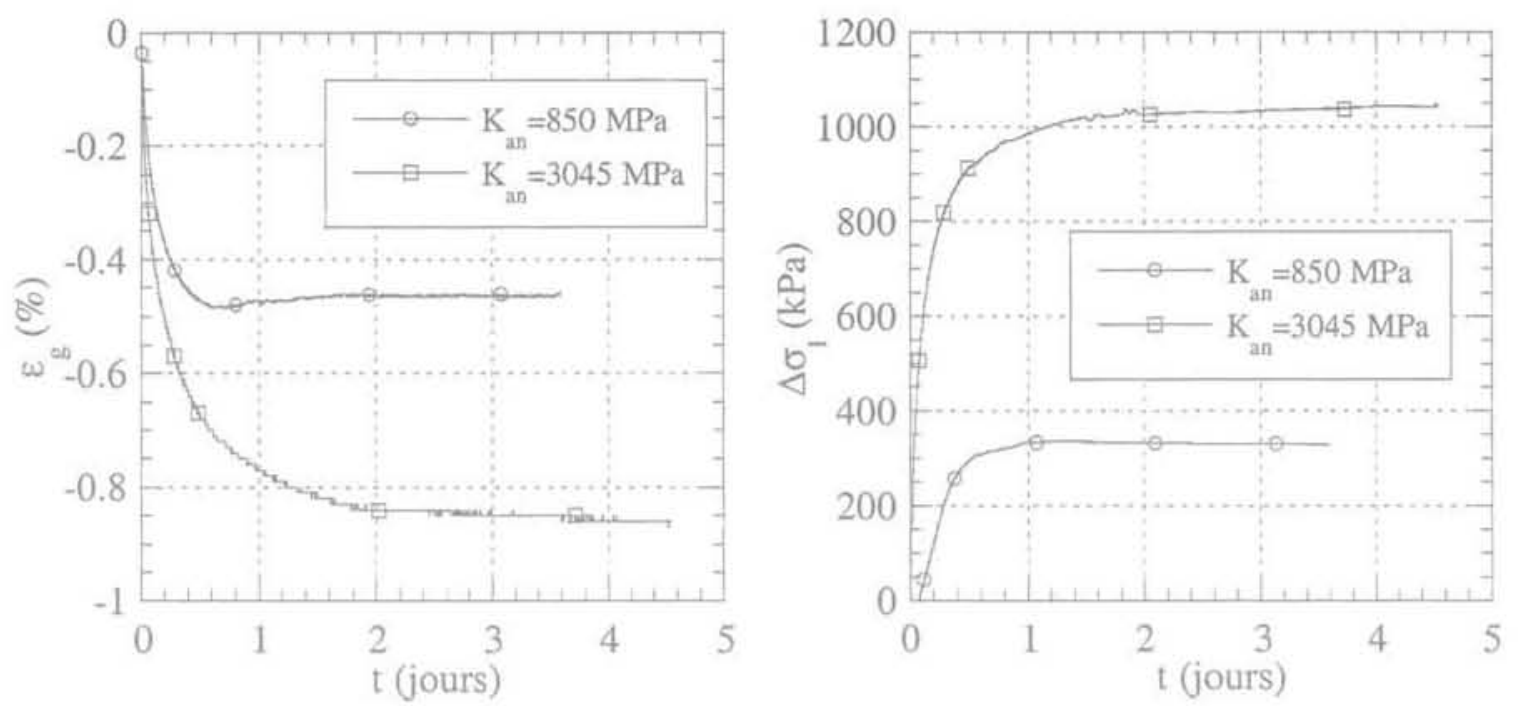

FG. 2 Influence de la rigiditẻ d'anneau sur le gonflement des éprouvettes non stratifiées. Phase d'imbibition sous $\sigma_{i}=780 \mathrm{kPa}$.

(a) Déformation de gonflement axiale ; (b) pression de gonflement latérale.

Influence of the ring stiffriess on the swelling of non-stratified specimens, Imbibition phase under $\sigma_{k}=780 \mathrm{kPa}$.

(a) Axial swelling strain; (b) latera! swelling pressure.
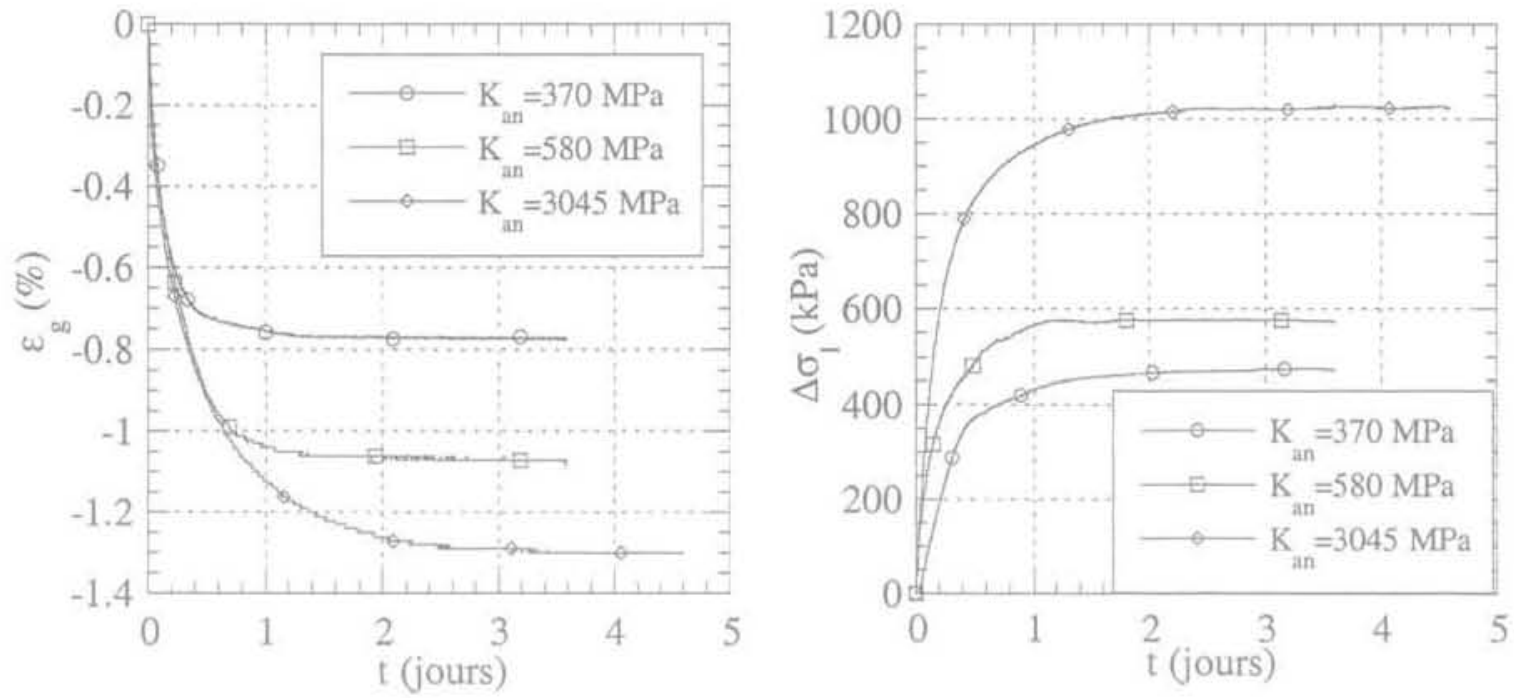

FG.3 Influence de la rigidité d'anneau sur le gonflement des éprouvettes stratifiées. Phase d'imbibition sous $\sigma_{a}$ $=780 \mathrm{kPa}$.

(a) Déformation de gonflement axiale ; (b) pression de gonflement latérale.

Influence of the ring stiffness on the swelling of stratified specimens. Imbibition phase under $\sigma_{\mathrm{a}}=780 \mathrm{kPa}$.

(a) Axial swelling strain; (b) lateral swelling pressure.

(D-E), (E-F) correspondent respectivement au cycle de chargement, déchargement, rechargement, à la phase d'imbibition et à la décharge par palier. En admettant que l'on puisse comparer les courbes (CD) et (EF), c'est-

TABLEAU II Déformation axiale et pression latérale induites pendant la phase d'imbibition sous une contrainte axiale $\sigma_{\mathrm{a} 0}=780 \mathrm{kPa}$. Axial deformation and lateral pressure induced by swelling during the imbibition phase under an axial stress $\sigma_{00}=780 \mathrm{kPa}$.

\begin{tabular}{l|ccc|cc} 
& \multicolumn{3}{|c|}{$\begin{array}{c}\text { Epprouvettes } \\
\text { stratifiées }\end{array}$} & $\begin{array}{c}\text { Éprouvettes } \\
\text { non stratifiées }\end{array}$ \\
$\mathrm{K}_{\text {un }}(\mathrm{MPa})$ & 370 & 580 & 3045 & 850 & 3045 \\
$\varepsilon_{\mathrm{g}}(\%)$ & $-0,77$ & $-1,09$ & $-1,30$ & $-0,46$ & $-0,86$ \\
$\Delta \sigma_{1}(\mathrm{kPa})$ & 473 & 573 & 1024 & 329 & 1043 \\
$q=\sigma_{\text {u }}-\sigma_{1}(\mathrm{kPa})$ & 230 & 154 & -258 & 426 & -277 \\
\hline
\end{tabular}

à-dire en supposant qu'il n'y ait pas d'évolution de la structure du matériau pendant le gonflement, on détermine la déformation axiale de gonflement hydrique sous une contrainte axiale $\sigma$ en retranchant de la déformation totale la déformation élastique due à la décharge mécanique, qui est calculée à partir du cycle de charge sur le matériau non imbibé. On trace alors dans le plan $\left(\log \sigma_{a}, \varepsilon_{\mathrm{o}}\right)$ la déformation axiale de gonflement hydrique en fonction de la contrainte axiale (Fig. 4d). On constate que les résultats peuvent être décrits par la relation logarithmique

$$
\varepsilon_{\mathrm{g}}=\mathrm{K}_{\mathrm{j}} \log \left(\frac{\sigma_{\mathrm{g}}}{\sigma_{\mathrm{g}}}\right)
$$

où $\mathrm{K}$ et $\sigma_{\mathrm{g}}$ désignent respectivement l'indice de gonflement et la pression de gonflement. Pour l'essai réalisé, on trouve $\mathrm{K}_{1}=0,0271$ et $\sigma_{\mathrm{a}}=1830 \mathrm{kPa}$. 


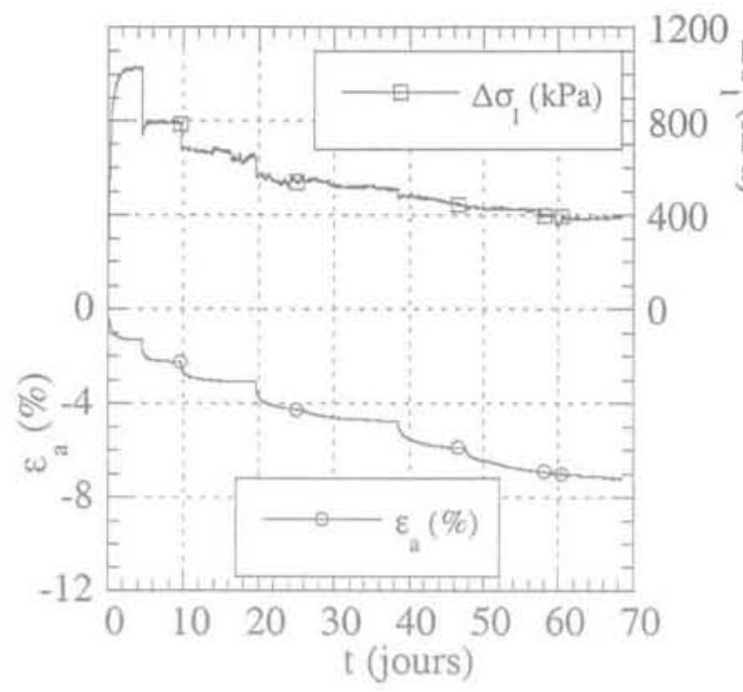

(a)

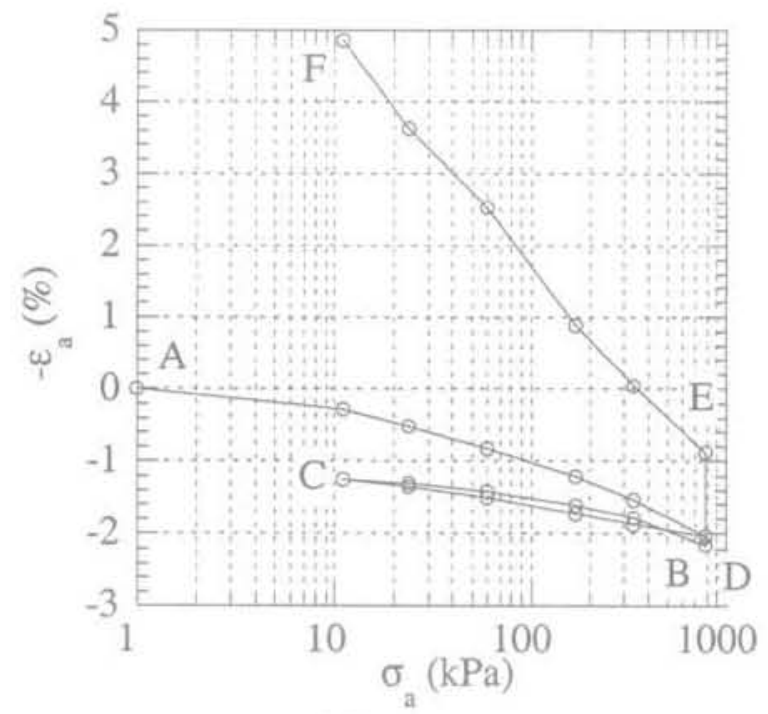

(c)

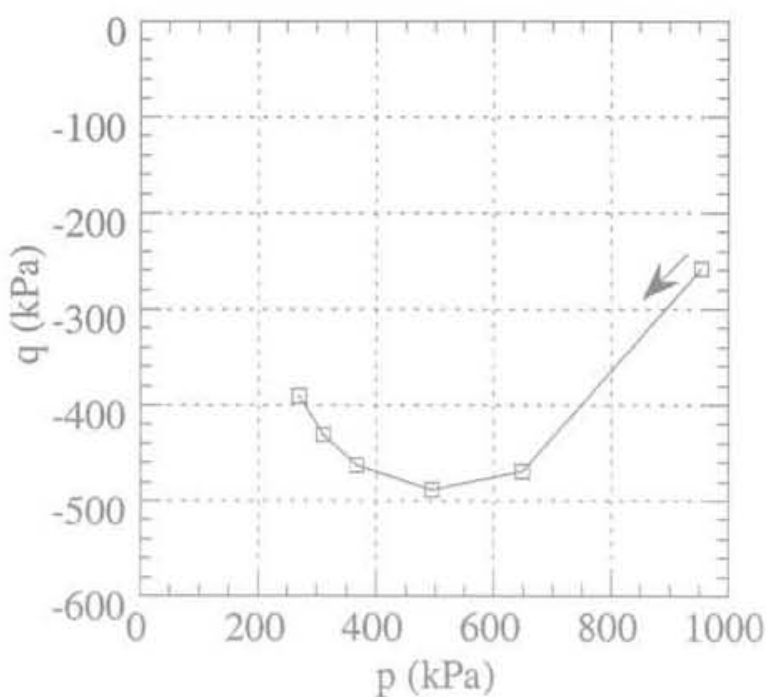

(b)

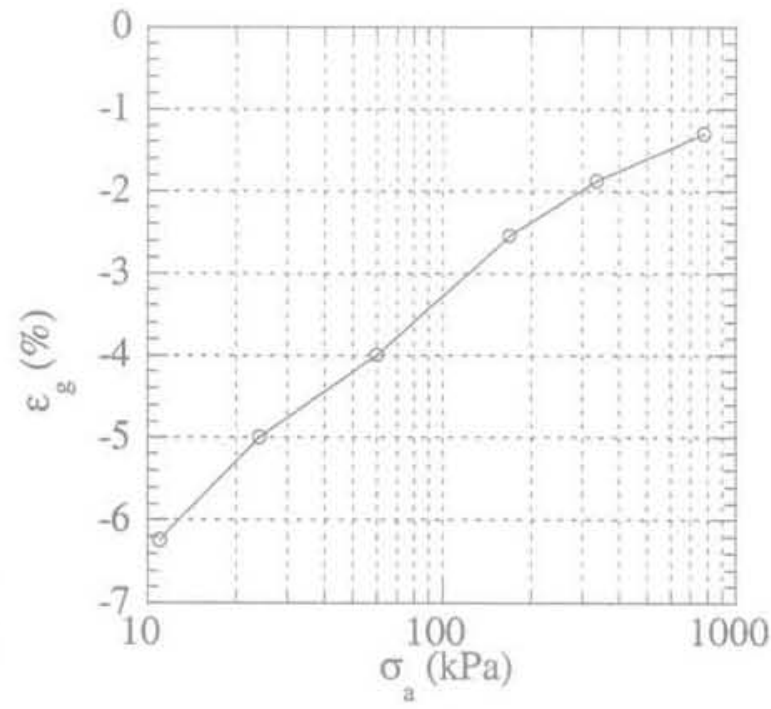

(d)

FG, 4 Essai de gonflement réalisé avec l'anneau de rigidité $\mathrm{K}_{\mathrm{n}}=3045 \mathrm{MPa}$ sur éprouvette stratifiée.

(a) Evolution de la déformation axiale et de la pression latérale pendant la phase de déchargement.

(b) Chemin de contrainte pendant la phase de déchargement.

(c) Évolution de la déformation axiale pendant les différentes phases de chargement.

(d) Évolution de la déformation axiale de gonflement pendant la phase de déchargement.

Swelling tests performed with the ring stiffness $K_{n}=3,045 \mathrm{MPa}$ on stratified specimen.

(a) Evolution of the axial strain and the lateral pressure during the unloading phase.

(b) Stress path during the unloading phase.

(c) Evolution of the axial strain during the various loading phase.

(d) Evolution of the axial swelling strain during the unloading phase.

La figure 5 donne l'influence de la rigidité de l'anneau et de la stratification sur le gonflement axial. On constate que l'augmentation de la rigidité de l'anneau a pour effet d'augmenter le gonflement axial. Le tableau IV donne les valeurs de l'indice de gonflement $\left(K_{0}\right)$ et de la pression de gonflement $\left(\sigma_{n}\right)$ pour les différents essais, On peut constater que les pressions de gonflement obtenues avec les éprouvettes stratifiées sont supérieures à celles des éprouvettes non stratifiées. En effet, la pression de gonflement axiale de la marne stratifiée varie entre 1830 et $1990 \mathrm{kPa}$ tandis qu'elle varie entre 1015 et $1125 \mathrm{kPa}$ pour la marne non stratifiée. L'indice de gonflement $\mathrm{K}$ augmente avec l'augmentation de la rigidité de la cellule. II varie de 0,0157 à 0,0271 lorsque la rigidité de l'anneau croît de 370 à $3045 \mathrm{MPa}$ pour les éprouvettes stratifiées et de 0,0217 à 0,0283 lorsque la rigidité de l'anneau augmente de 850 à $3045 \mathrm{MPa}$ pour les éprouvettes non stratifiées.

Le potentiel de gonflement est généralement caractérisé par le couple $\left(\sigma_{\sigma}, \mathrm{K}\right)$. Afin de comparer ces deux paramètres simultanément, Bultel (2001) a défini le paramètre $k_{\text {g }}$ qui représente l'amplitude de la déformation axiale de gonflement lorsque la contrainte axiale diminue de $\sigma_{\mathrm{g}}$ à $1 \mathrm{kPa}$ :

$$
k_{0}=K_{i} \log \left(\sigma_{g}\right)
$$


TABLEAUIII Évolution de la contrainte latérale et du déviateur pendant la phase de déchargement - essai réalisé sur l'éprouvette stratifiée avec l'anneau de rigidité $\mathrm{K}_{\mathrm{n}}=3045 \mathrm{MPa}$, contrainte axiale initiale $\sigma_{\mathrm{n0}}=780 \mathrm{kPa}$. Evolution of lateral pressure and deviator stress during the imbibition phase - test carried out on the stratified specimen with the ring stiffness $\mathrm{K}_{m}=3,045 \mathrm{MPa}$, initial axial stress $\sigma_{m n}=780 \mathrm{kPa}$.

\begin{tabular}{lcccc}
\hline Palier & $\Delta \sigma_{2}(\mathrm{kPa})$ & $\Delta \sigma_{1}(\mathrm{kPa})$ & $\Delta \sigma_{1} / \Delta \sigma_{\mathrm{a}}$ & $q=\sigma_{\mathrm{i}}-\sigma_{1}(\mathrm{kPa})$ \\
\hline 1 & -448 & -237 & 0,52 & -469 \\
2 & -165 & -146 & 0,89 & -488 \\
3 & -110 & -136 & 1,24 & -462 \\
4 & -36 & -67 & 1,88 & -432 \\
5 & -13 & -54 & 4,01 & -390 \\
\hline
\end{tabular}

Les valeurs de $k_{f}$ sont également reportées dans le tableau IV. On note que l'augmentation de la rigidité de la cellule a tendance à faire augmenter ce facteur. Si l'on considère ce paramètre comme un indicateur du potentiel de gonflement du sol dans la direction axiale, ces résultats montrent que le fait d'empêcher le gonflement dans la direction latérale amplifie le potentiel de gonflement dans la direction axiale.

La figure 6 illustre l'évolution de la contrainte latérale au cours de la phase de déchargement. On note que l'évolution de cette contrainte dépend de la rigidité de l'anneau. Le tableau V donne le rapport entre les variations de la pression latérale et de la contrainte axiale pour les différents paliers de déchargement (???).

$$
\left(R_{\mathrm{la}}=\frac{\Delta \sigma_{1}}{\Delta \sigma_{\mathrm{a}}}\right)
$$

On note que ce rapport dépend de la rigidité de la cellule et de la contrainte axiale. En effet, il augmente avec la diminution de contrainte axiale et avec l'augmentation de la rigidité de la cellule. Pour les éprouvettes non stratifiées, ce rapport varie de 0,22 à 0,96 pour l'essai réalisé avec l'anneau de rigidité $\mathrm{K}_{\mathrm{an}}=850 \mathrm{MPa}$ et entre 0,73 et 1,73 pour l'essai réalisé avec l'anneau de rigidité $\mathrm{K}_{u}=$ $3045 \mathrm{MPa}$. Les faibles valeurs de ce rapport au cours des premiers paliers de déchargement donnent une réduction du déviateur de contrainte $(q=\sigma-\sigma)$, ce qui a pour conséquence de conduire les éprouvettes qui étaient à la

\begin{tabular}{|c|c|c|c|c|c|}
\hline TABLEAU IV & \multicolumn{5}{|c|}{$\begin{array}{l}\text { Paramètres de gonflement } \\
\text { dans la direction axiale déterminés } \\
\text { à partir de la phase de déchargement. } \\
\text { Axial swelling parameter obtained from the } \\
\text { unloading phase. }\end{array}$} \\
\hline $\mathrm{R}_{\mathrm{lp}}$ & \multicolumn{3}{|c|}{$\begin{array}{l}\text { Éprouvettes } \\
\text { stratifiées }\end{array}$} & \multicolumn{2}{|c|}{$\begin{array}{l}\text { Eiprouvettes } \\
\text { non stratifiees }\end{array}$} \\
\hline $\begin{array}{l}K_{\text {win }}(\mathrm{MPa}) \\
\mathrm{K}_{9}(\mathrm{KPa}) \\
\mathrm{K}_{g}(\%)\end{array}$ & $\begin{array}{r}370 \\
0,0157 \\
1990 \\
-5,18\end{array}$ & $\begin{array}{c}580 \\
0,0256 \\
1860 \\
-8,37\end{array}$ & $\begin{array}{r}3045 \\
0,0271 \\
1830 \\
-8,84\end{array}$ & $\begin{array}{c}850 \\
0,0217 \\
1015 \\
-6,52\end{array}$ & $\begin{array}{r}3045 \\
0,0283 \\
1125 \\
-8,63\end{array}$ \\
\hline
\end{tabular}

TABLEAUV Influence de la rigidité d'anneau sur la variation de la contrainte latérale induite au cours de la phase de déchargement. Influence of the ring stiffness on the variation of the lateral stress induced during the unloading phase.

\begin{tabular}{l|lll|ll|}
\hline $\mathrm{R}_{\mathrm{i}}$ & \multicolumn{3}{|c|}{$\begin{array}{c}\text { Éprouvettes } \\
\text { stratifiées }\end{array}$} & $\begin{array}{c}\text { Éprouvettes } \\
\text { non stratifiées }\end{array}$ \\
\hline K (MPa) & 370 & 580 & 3045 & 850 & 3045 \\
Palier 1 & 0,25 & 0,45 & 0,52 & 0,22 & 0,73 \\
Palier 2 & 0,34 & 0,71 & 0,89 & 0,35 & 0,93 \\
Palier 3 & 0,53 & 1,12 & 1,24 & 0,41 & 1,17 \\
Palier 4 & 1,32 & 1,47 & 1,88 & 0,67 & 1,73 \\
Palier 5 & 2,35 & 2,56 & 4,01 & 0,96 & 1,01 \\
\hline
\end{tabular}
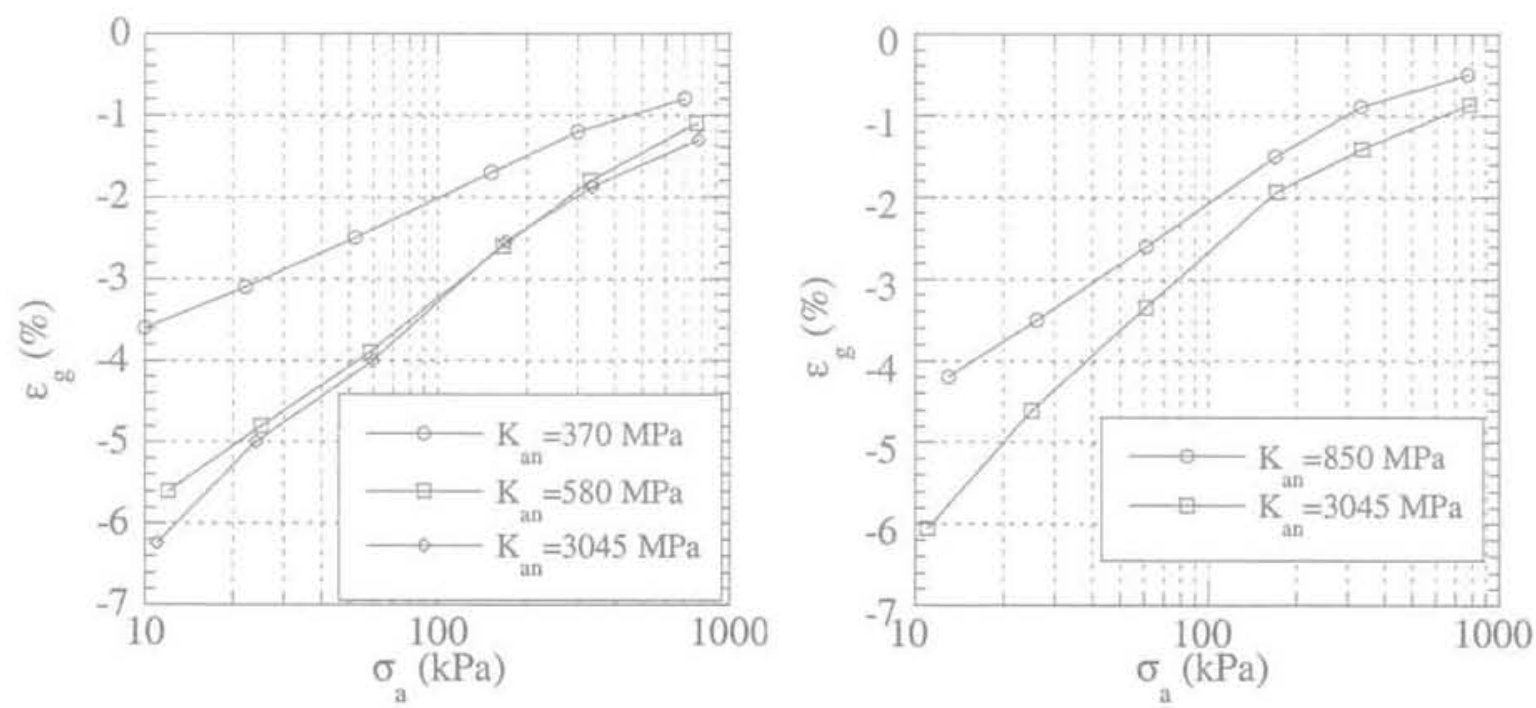

FIG. 5 Influence de la rigidité d'anneau sur la déformation axiale de gonflement pendant la phase de déchargement.

(a) Éprouvettes stratifiées ; (b) éprouvettes non stratifiées.

Influence of the ring stiffness on the axial swelling strain during the unloading phase.

(a) Stratified specimens; (b) non-stratified specimens 

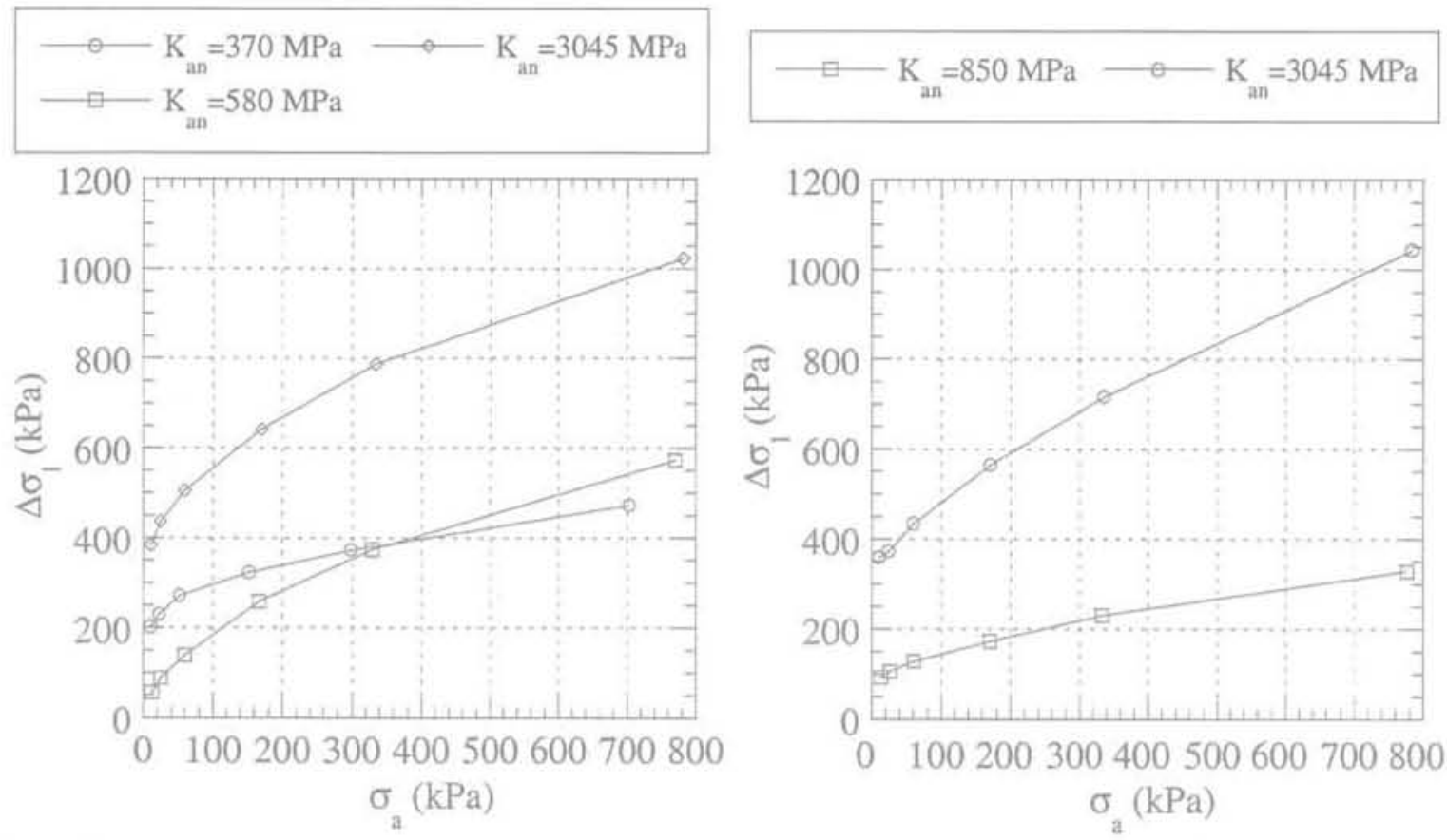

FG.6 Influence de la rigidité d'anneau sur la pression latérale pendant la phase de déchargement.

(a) Éprouvettes stratifiées ; (b) éprouvettes non stratifiées.

Influence of the ring stiffness on the lateral pressure during the unloading phase.

(a) Stratified specimens; (b) non-stratified specimens.

fin de la phase d'imbibition dans un état de compression à un état d'extension et à accentuer l'état d'extension des éprouvettes qui l'étaient déjà (Fig. 7). Au cours des derniers paliers, on note généralement des valeurs de $R_{t}$ supérieures à 1 , ce qui signifie que la contrainte latérale décroît moins que la contrainte axiale. Ceci correspond à une augmentation du déviateur, et par conséquent, à une réduction de l'état d'extension. Les derniers paliers de déchargement correspondent à une réduction impor- tante de la contrainte moyenne, ce qui conduit une bonne partie des éprouvettes à la plasticité (Fig. 7).

\section{3}

\section{Anisotropie de gonflement}

On propose dans cette section d'étudier l'influence de la stratification sur l'anisotropie de la pression de
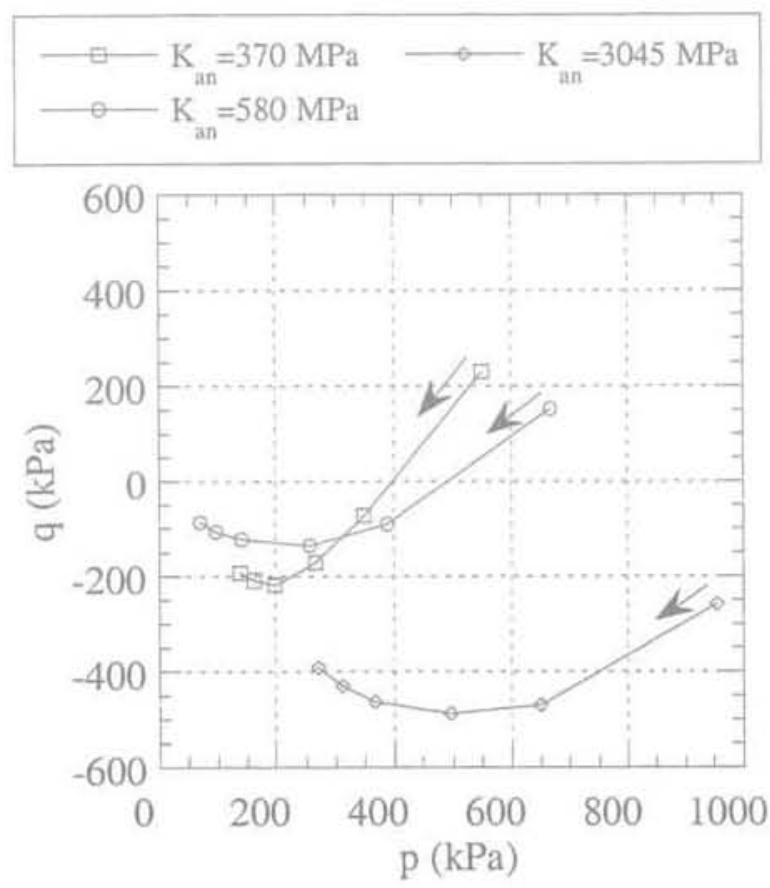
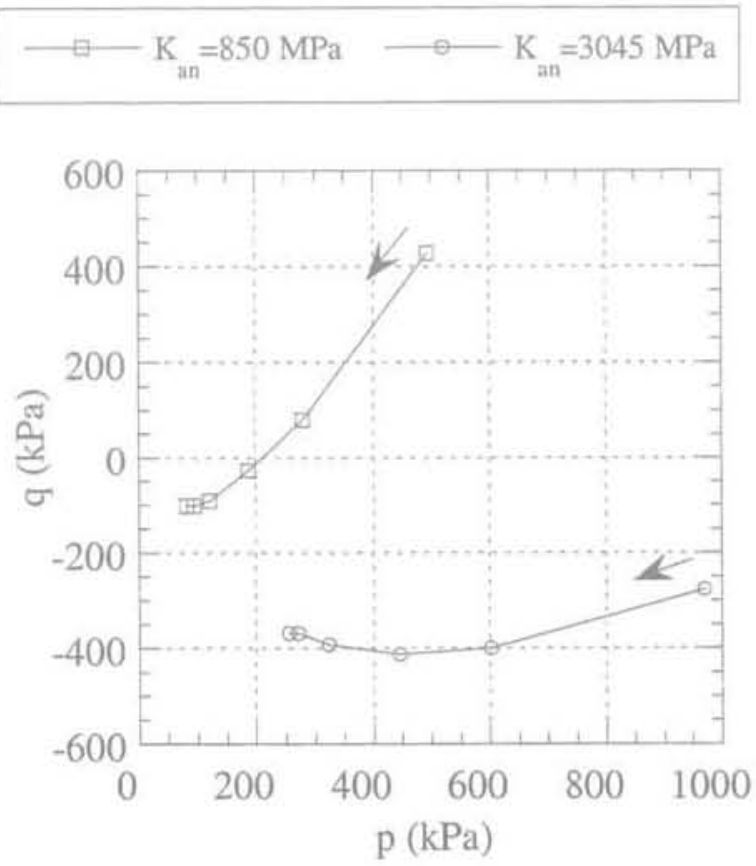

FIG.7 Influence de la rigidité d'anneau sur le chemin de contrainte dans le plan (p, q) induit par le gonflement pendant la phase de déchargement.

(a) Éprouvettes stratifiées; (b) éprouvettes non stratifiées.

Influence of the ring stiffness on the stress path in ( $\mathrm{p}$, q) plan induced by swelling during the unloading phase.

(a) Stratified specimens; (b) non-stratifled specimens. 
gonflement. Dans un premier temps, on détermine les pressions de gonflement latérale et axiale pour les éprouvettes non stratifiées, ensuite on les étudie pour les éprouvettes stratifiées.

Du fait que la pression de gonflement dépend du niveau de contrainte et des conditions de déformations, on utilise l'essai réalisé avec l'anneau de rigidité élevée $\left(\mathrm{K}_{\mathrm{an}}=3045 \mathrm{MPa}\right)$ pour déterminer la pression de gonflement des éprouvettes non stratifiées. En effet, lors de la phase d'imbibition de cet essai, la déformation axiale est fortement empêchée par la contrainte axiale relativement élevée $\left(\sigma_{0}=780 \mathrm{kPa}\right)$, et lors de la phase de décharge, la rigidité élevée de l'anneau empêche le développement de la déformation radiale. On obtient pour cet essai une pression de gonflement latérale de $1043 \mathrm{kPa}$ et une pression de gonflement axiale de 1125 $\mathrm{kPa}$. Ces deux pressions étant relativement proches, on peut considérer que l'éprouvette développe une pression de gonflement isotrope. Le caractère isotrope de la pression de gonflement a été confirmé par un essai à déformation axiale bloquée, qui a été réalisé avec l'anneau de rigidité $\mathrm{K}_{\mathrm{nn}}=3045 \mathrm{MPa}$ monté dans un bâti de très grande rigidité. Pour cet essai, les conditions aux limites imposées à l'éprouvette dans la direction axiale et radiale sont très proches, car les rigidités du bâti et de la cellule empêchent l'éprouvette de gonfler. On présente sur la figure 8 l'évolution des contraintes axiale et. radiale au cours de cet essai. On constate que ces pressions évoluent d'une manière identique et conduisent à une pression de gonflement égale à $860 \mathrm{kPa}$. On peut noter que cette pression est inférieure de $20 \%$ à celle déterminée à l'aide de l'essai d'Huder-Amberg. Ce résultat est en accord avec les résultats obtenus par Khaddaj (1992), qui ont montré que la méthode directe donne une pression de gonflement inférieure à celles données par la méthode de gonflement en parallèle et la méthode d'Huder-Amberg.

La démarche suivie ci-dessus est appliquée à l'éprouvette stratifiée. L'essai réalisé avec l'anneau de rigidité $\mathrm{K}_{\mathrm{an}}=3045 \mathrm{MPa}$ donne une pression de gonflement axiale de $1830 \mathrm{MPa}$ (tableau IV) qui est supérieure de $80 \%$ à la pression de gonflement latérale. L'anisotropie

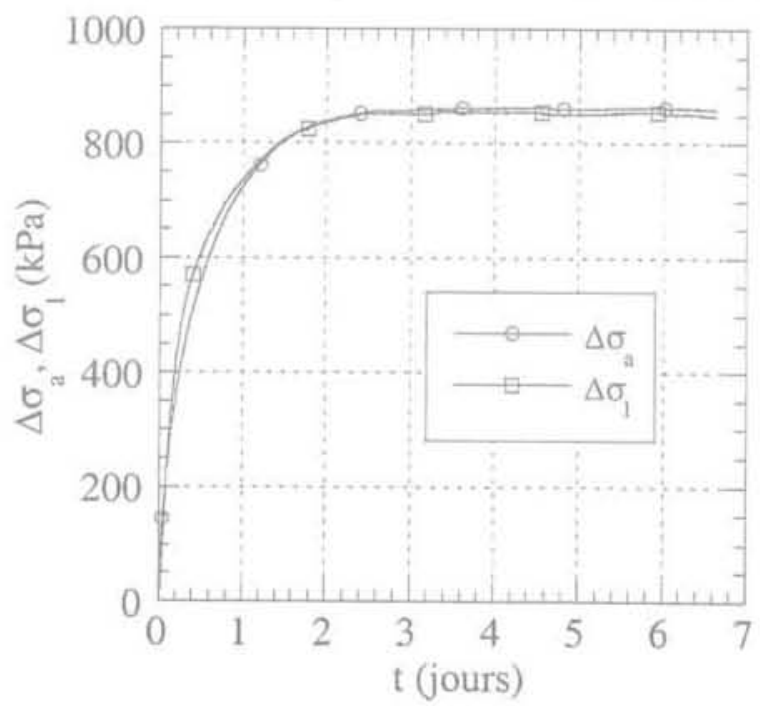

FG. B Essai de gonflement à déformation axiale bloquée réalisé avec l'anneau de rigidité $\mathrm{K}_{\mathrm{an}}=3045 \mathrm{MPa}$ sur une éprouvette non stratifiée.

Swelling test with blocked axial strain carried out with a ring stiffness $\mathrm{K}_{-}=3,045 \mathrm{MPa}$ on a non-stratified specimen. de gonflement des éprouvettes stratifiées est confirmée par un essai de gonflement libre sur une éprouvette stratifiée, avec mesure des déformations radiale et axiale (Fig. 9). Le rapport entre la déformation axiale et la déformation latérale atteint 4,3 en fin d'imbibition.

\section{5}

\section{Conclusion}

L'œdomètre flexible permet de réaliser des essais de gonflement particulièrement intéressants pour les projets de tunnels. En effet, en faisant varier la rigidité de l'anneau, on peut réaliser des essais de gonflement sur des chemins de chargement allant de l'essai de gonflement libre à l'essai de gonflement à l'cedomètre classique. On peut ainsi étudier l'influence d'une possibilité de déformation des sols autour des tunnels sur leur gonflement. Cet appareillage offre également la possibilité de mesurer la contrainte latérale, ce qui permet de disposer de données intéressantes pour la validation et le développement des modèles tridimensionnels de gonflement.

Les résultats des essais réalisés avec cet appareillage sur des marnes ( intacts » ont mis en évidence une forte interaction entre la condition de chargement dans la direction latérale et les propriétés de gonflement. L'autorisation d'une déformation latérale induit une diminution de la pression de gonflement latêrale et de la déformation axiale de gonflement. Ceci indique que les essais réalisés avec l'œdomètre classique tendent à surestimer la pression de gonflement dans la direction axiale dans le cas où le sol in situ peut développer une déformation au cours du processus de gonflement.

Les essais réalisés sur des éprouvettes stratifiées ont montré que le gonflement se développe principalement dans la direction perpendiculaire à la stratification donnant ainsi une forte anisotropie de gonflement. Par contre, pour des éprouvettes non stratifiées, on observe un gonflement isotrope.

Cette étude apporte des données intéressantes sur le comportement tridimensionnel des sols gonflants qui pourront être utilisées par la suite pour la validation ou l'adaptation de modèles existants, ou encore pour le développement de nouveaux modèles pour le calcul de tunnels concernés par les terrains gonflants.

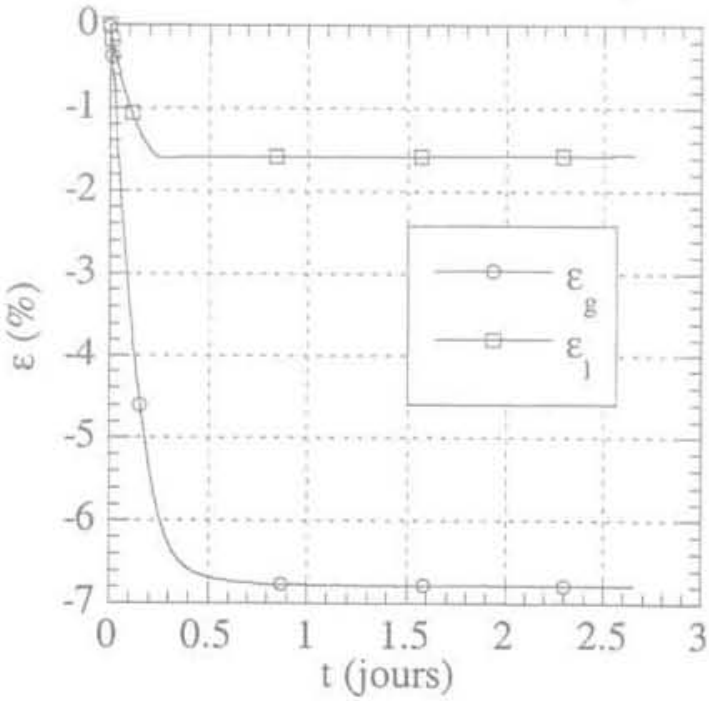

FG.9 Essai de gonflement libre réalisé sur une éprouvette stratifiée $\left(\sigma_{a 0}=10 \mathrm{kPa}\right)$. Free swelling test carried out on a stratified specimen $\left(\sigma_{30}=10 \mathrm{kPa}\right)$. 
Bultel F, - Prise en compte du gonflement des terrains pour le dimensionnement des revêtements des tunnels. Thèse de doctorat. Ecole nationale des ponts et chaussées, Paris, 2001

Einstein H.H. Bischoff N, $-\alpha$ Dimensionnement des tunnels en roche gonflante x. Tunnels et Ouvrages souterrains, $n^{\circ} 15,1976$, p. 109-119.

Froehlich B.O. - * Géologie et constructions des tunnels : gonflement de roches argileuses y. Rock at areat depth. Maurv \& Fourmaintaux (eds), 1989, p. 537-544.

Holtz W.G.. Gibbs H.J. - « Engineering properties of expansive clays $n$. Transaction of ASCE, vol. 121, 1956, p. 641-663.

Kabbaj M. - « Sols gonflants : mesure des propriétés en laboratoire 1. Revue marocaine du qénie civil, n² 26, 1989, p. 17-27.

Khaddaj S. - Etude en laboratoire du gon- flement de l'argile des Flandres. Thèse de I'Université des sciences et technologies de Lille, 1992

Kiehl J.R. - u Ein dreidimensionales Quellgesetz und seine Anwendung auf den Felshohlraumbau ग. 9th National Rock Mechanics Symposium. Aachen Germany, 1990, p. 185-207.

Kovari K. Amstad C. Anagnostou G. * Long term field observations of swelling phenomena in tunnel and their back analysis b. Colloquium Mundanum. 1995.

Robert A. - Construire sur les sols gonflants et rétractables. Ecole nationale des ponts et chaussées, Formation continue, 1991.

Seed H.B.. Mitchell J.K., Chan C.K. - " Stu. dies of swell and swell pressure characteristics of compacted clays \%. Higway Research Board, vol. 313, 1962, p. 12-39.
Serratrice 1.F. - Barreau B55 - Liaison A55/A7 - Essais de laboratoire. Rapport du Laboratoire régional des ponts et chaussées d'Aix-en-Provence, ifs Barreau B55, 1998.

Steiner W. $-\pi$ Swelling rock in tunnels : rock characterization, effect of horizontal stresses and construction procedures in. International Journal of Rock Mechanics and Mining Sciences and Geomechanics Abstracts, vol. $30, n^{\circ} 4,1993$, p. $361-380$.

Windal T. - Etude en laboratoire du gonflement des sols : mise au point d'un cedométre flexible et étude du gonflement tridimensionnel. Thèse de l'Universitê des sciences et technologies de Lille, 2001.

Wittke W. Pierau B. - « Fundamentals for the design and construction of tunnels in swelling rock 1. 4th ICRM, Montreux, vol. 2,1979 , p. $719-729$. 